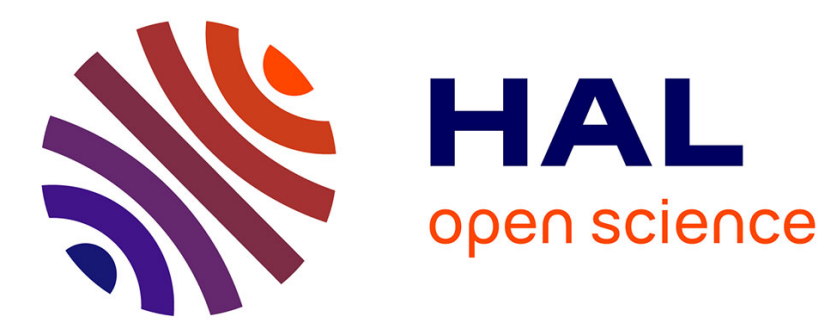

\title{
Dynamics of networks in a viscoelastic and active environment
}

\author{
Jonas Grimm, Maxim Dolgushev
}

\section{To cite this version:}

Jonas Grimm, Maxim Dolgushev. Dynamics of networks in a viscoelastic and active environment. Soft Matter, 2018, 14 (7), pp.1171-1180. 10.1039/C7SM02050C . hal-02189250

\section{HAL Id: hal-02189250 \\ https://hal.sorbonne-universite.fr/hal-02189250}

Submitted on 19 Jul 2019

HAL is a multi-disciplinary open access archive for the deposit and dissemination of scientific research documents, whether they are published or not. The documents may come from teaching and research institutions in France or abroad, or from public or private research centers.
L'archive ouverte pluridisciplinaire HAL, est destinée au dépôt et à la diffusion de documents scientifiques de niveau recherche, publiés ou non, émanant des établissements d'enseignement et de recherche français ou étrangers, des laboratoires publics ou privés. 


\title{
Dynamics of networks in a viscoelastic and active en- vironment
}

\author{
Jonas Grimm ${ }^{a}$ and Maxim Dolgushev ${ }^{* a b}$
}

\begin{abstract}
We investigate the dynamics of fractals and other networks in a viscoelastic and active environment. The viscoelastic dynamics is modeled based on the generalized Langevin equation, where the activity is introduced to it by means of the exponentially correlated noise. The intramolecular interactions are taken into account by the bead-spring picture. The microscopic connectivity (studied in form of Viscek fractals, of dual Sierpiński gaskets, of $N T_{D}$ trees, and of a family of deterministic small-world networks) reveals itself in the multiscale monomeric dynamics, which shows vastly different behaviors in the active and passive baths. In particular, the dynamics under active forces leads to a swelling that is characterized through power laws which are not present in the passive case. In all cases the dynamics reflects the broad scaling behavior of the density of states and not necessarily the maximal relaxation time of the structures in a passive bath, as it is exemplified on the $N T_{D}$ trees.
\end{abstract}

\section{Introduction}

Modern experiments on the dynamics of biopolymers in a crowded active bath (such as motion of chromosomal loci in bacteria) have demonstrated striking features of the macromolecular systems in the out-of-equilibrium environments. ${ }^{112}$ Indeed, as has been shown in a rapidly growing series of recent studies,, $3-16$ the behavior of macromolecules in the active and crowded environments differs tremendously from that of the polymers in a usual thermal bath.17 In particular, the activity leads typically to a swelling of macromolecules, $\frac{5,8}{5}$ the dynamics is drastically enhanced yielding a change of scalings, 4 , $10 \mid 11$ and the kinetics of the intramolecular reactions is facilitated. .7

It is necessary to note that the structure of biomacromolecules is typically very complex. In this respect, fractal and network models provide a powerful approach to these complex objects. ${ }^{18}+23$ From the practical side, deterministic structures with quenched connectivity allow for analytic calculations, based on which the scaling behavior of very large objects can be studied. 24.28

The analysis of tracking experiments of chromosomal loci in live bacterial cells has favored the generalized Langevin equation approach for modeling the viscoelasticity of bacterial environment. ${ }^{1}$ Moreover, the activity of the environment is commonly modeled by exponentially correlated noise $\mathrm{3}^{3 / 4|10| 12[14 / 15}$ that is

\footnotetext{
a Institute of Physics, University of Freiburg, Hermann-Herder-Str.3, D-79104 Freiburg, Germany.E-mail: dolgushev@physik.uni-freiburg.de

${ }^{b}$ Laboratoire de Physique Théorique de la Matière Condensée, CNRS UMR 7600, Université Pierre-et-Marie-Curie, 4 Place Jussieu, 75005 Paris, France.

E-mail: dolgushev@lptmc.jussieu.fr
}

also found in the analysis of experimental data. ${ }^{29}$ Recently, the behavior of a bead-spring linear chain in an active and vicsoelastic bath has been studied. ${ }^{410|14| 15}$ Sakaue and Saito ${ }^{12}$ did a scaling generalization of the results for linear chains to fractals. In the present work we study fractals and more general networks based on the microscopic connectivity. This approach allows us to monitor explicitly the microscopic dynamics and to investigate its influence on the macroscopic behavior. In particular, we study treelike fractals (Vicsek fractals ${ }^{25}$ ) and fractals with loops (dual Sierpiński gaskets ${ }^{24}$ ), which both are rather homogeneous structures, the so-called $N T_{D}$ trees ${ }^{30+32}$ that posses a heterogeneous density of states, and the small-world networks (SWNs) of ref. ${ }^{33}$ whose density of states is characterized by the terminal value of the spectral dimension $d_{s}=2$. We show that in case of structurally homogeneous fractals the connectivity is clearly reflected in the monomer displacement through a region characterized by the corresponding spectral dimension $d_{s}$, in agreement with the generalized scaling argument of Sakaue and Saito. ${ }^{[12}$ For the $N T_{D}$ trees, whose maximal relaxation time (for the parameters used in the paper) behaves in a passive viscous bath as $N^{1}$, the scalings of linear chains are observed (albeit the maximal relaxation time of a linear chain in a passive viscous bath is proportional to $N^{2}$ ). The SWNs, that under passive conditions are in a collapsed state so that the monomers have a logarithmically slow motion, under active forces display a much faster, anomalous monomer dynamics.

The paper is structured as follows: Section 2 is devoted to the framework of generalized Langevin equation and to the characterization of macromolecular networks studied in the paper. The 
dynamical behavior of the networks is discussed in Section 3 . The paper closes with the conclusions presented in Section 4

\section{Theoretical Methods}

\subsection{The model}

We start with a brief description of the model used in the paper, see also its schematic representation in Figure 1

The macromolecular structures are modeled as $N$ beads connected by springs. The connectivity is encoded by the Laplacian matrix $\mathbf{A}=\left(A_{n m}\right) \cdot{ }^{\sqrt[34]{ }}$ Its diagonal elements read $A_{n n}=f_{n}$, where $f_{n}$ is the functionality of the $n$th bead (i.e., number of nearest neighbors of the $n$th bead). The other elements are $A_{n m}=A_{m n}=-1$, if beads $n$ and $m$ are directly connected, and $A_{n m}=A_{m n}=0$ else.

The dynamics of such a macromolecule is described by the overdamped generalized Langevin equation. 35 Here the beads located in a three-dimensional space at $\vec{R}_{n}(t)(n=1,2, \ldots, N)$ and connected by harmonic springs are put in a viscoelastic and active bath:

$$
\gamma \int_{0}^{t} \mathrm{~d} \tau K(t-\tau) \dot{\vec{R}}_{n}(\tau)=-k \sum_{m=1}^{N} A_{n m} \vec{R}_{m}(t)+\vec{\xi}_{T, n}(t)+\vec{\xi}_{A, n}(t) H(t),
$$

where $\gamma$ is the friction coefficient, $k$ is the spring constant and $H(t)$ denotes the Heaviside function. Moreover, $K(t)$ is a power law kernel which takes into account the memory of the friction force due to the crowded viscoelastic environment,

$$
K(t)=(2-\alpha)(1-\alpha) t^{-\alpha} .
$$

With this, due to the fluctuation-dissipation theorem, 40 the behavior of random thermal forces $\left\{\vec{\xi}_{T, n}(t)\right\}$ reads

$$
\left\langle\vec{\xi}_{T, n}(t) \vec{\xi}_{T, m}\left(t^{\prime}\right)\right\rangle=3 \gamma k_{B} T K\left(\left|t-t^{\prime}\right|\right) \delta_{n, m},
$$

where $T$ is the temperature and $k_{B}$ is the Boltzmann constant. The thermal forces have vanishing mean value and are Gaussian distributed. We notice that for $\alpha \rightarrow 1$ we have $K(t) \rightarrow \delta(t)$ (i.e., white noise), which reflects viscous behavior, while $\alpha=0$ implies elastic behavior. For $0<\alpha<1$, which is often observed in experiments, 141,42 the bath is vicsoelastic. Additionally to the viscoelastic bath, the system is driven, starting at time $t=0$, to the out-of-equilibrium state by the active forces $\vec{\xi}_{A, n}(t)$. These forces are taken to be Gaussian distributed with zero mean and the second moment

$$
\left\langle\vec{\xi}_{A, n}(t) \vec{\xi}_{A, m}\left(t^{\prime}\right)\right\rangle=3 C \exp \left(-\left|t-t^{\prime}\right| / \tau_{A}\right) \delta_{n, m},
$$

where $C$ characterizes the strength of the active forces and $\tau_{A}$ characterizes the time scale of the correlation. Such shot-noise force correlations are typically observed in the biological systems. ${ }^{29}$ We note that, in general, driving a system from the equilibrium leads to a violation of the fluctuation-dissipation theorem, which is reflected in the absent contribution of the active forces to the friction term of eqn (1). Nevertheless, as has been recently shown in ref. 14 , the noise of eqn (4) preserves the generalized Langevin equation for a tagged monomer so that the total noise

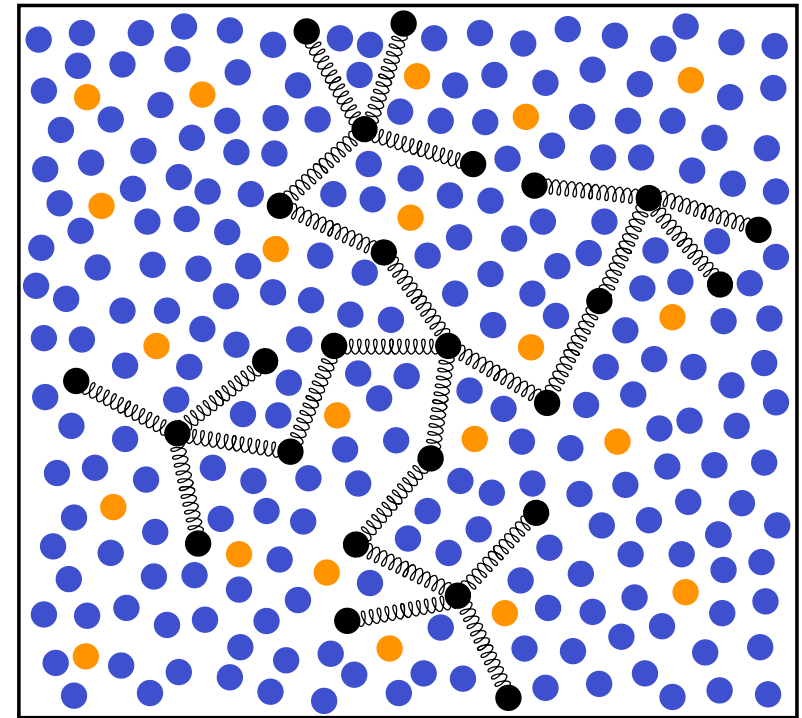

Fig. 1 Schematic representation of the problem studied in the paper: A macromolecule having complex connectivity in a dense environment of passive (blue) and active (orange) bath particles. Note that the spatial conformations of the macromolecule in a three-dimensional space may come in vastly different forms.

remains to be a centered Gaussian process.

Now, eqn (1) can be rewritten based on fractional derivatives in the Caputo sense 43

$$
{ }_{c} D^{\alpha} x(t)=I^{m-\alpha} x^{(m)}(t)
$$

where

$$
I^{\mu} x(t)=\frac{1}{\Gamma(\mu)} \int_{0}^{t} \mathrm{~d} \tau(t-\tau)^{\mu-1} x(\tau)
$$

is the Riemann-Liouville fractional integral. With this, eqn (1) reads

$$
\gamma \Gamma(3-\alpha)_{c} D^{\alpha} \vec{R}_{n}(t)=-k \sum_{m=1}^{N} A_{n m} \vec{R}_{m}(t)+\vec{\xi}_{T, n}(t)+\vec{\xi}_{A, n}(t) H(t) .
$$

In order to decouple this set of equations we introduce normal modes,

$$
\vec{X}_{p}(t)=\sum_{n=1}^{N} \vec{R}_{n}(t) Q_{n p}
$$

where $p=0,1, \ldots, N-1$ and $\mathbf{Q}$ is the matrix that diagonalizes $\mathbf{A}$ (i.e., $\mathbf{Q}^{-1} \mathbf{A} \mathbf{Q}=\operatorname{diag}\left(\lambda_{0}, \lambda_{1}, \ldots, \lambda_{N-1}\right)$, with $\lambda_{0}=0$ ). With eqn (8) we obtain

$$
\Gamma(3-\alpha)_{c} D^{\alpha} \vec{X}_{p}(t)=-\frac{k \lambda_{p} \vec{X}_{p}(t)}{\gamma}+\frac{\vec{\xi}_{p}(t)}{\gamma}
$$

with

$$
\vec{\xi}_{p}(t)=\sum_{n=1}^{N}\left(\vec{\xi}_{T, n}(t)+\vec{\xi}_{A, n}(t) H(t)\right) Q_{n p}
$$

Eqn (9) can be solved using Laplace-transform techniques. 43 
At time $t=0$ the system is in the thermal equilibrium, so that the initial condition is given by $\left\langle\vec{X}_{p}(0) \vec{X}_{q}(0)\right\rangle=\delta_{p q} l_{0}^{2} / \lambda_{p}$, where $l_{0}^{2}=3 k_{B} T / k$ is the mean squared length of the bond at time $t=0$. (Of course, one can think about out-of-equilibrium initial conditions, bearing in mind, e.g., in vivo experiments. In such a case the choice of the initial condition should be provided by the explicit experimental setup, which will then leave, due to the nonMarkovian monomer dynamics with non-stationary increments, its fingerprints in the evolution of the macromolecule's conformation.) Eqn (9)-(10) lead, in line with the results of ref. ${ }^{4}$ for a linear chain, to the correlation functions

$$
\begin{aligned}
\left\langle\left(\vec{X}_{0}(t)-\vec{X}_{0}(0)\right)^{2}\right\rangle & =\frac{2 l_{0}^{2}}{\alpha \Gamma(\alpha)}\left(\frac{t}{\tau_{0}}\right)^{\alpha} \\
& +\frac{6 C \tau_{A}^{2 \alpha}}{\gamma^{2} G_{\alpha}^{2}} \int_{0}^{t / \tau_{A}} \mathrm{~d} y e^{y} y^{\alpha-1} \Gamma\left(\alpha ; y, \frac{t}{\tau_{A}}\right)
\end{aligned}
$$

with $G_{\alpha}=\Gamma(\alpha) \Gamma(3-\alpha)$, and for $p \neq 0$ or $q \neq 0$ to

$$
\left\langle\vec{X}_{p}(t) \vec{X}_{q}(0)\right\rangle=\frac{l_{0}^{2} \delta_{p q}}{\lambda_{p}} E_{\alpha, 1}\left(-\left(\frac{t}{\tau_{0}}\right)^{\alpha} \lambda_{p}\right)
$$

and

$$
\begin{aligned}
\left\langle\vec{X}_{p}(t) \vec{X}_{q}(t)\right\rangle & =\frac{l_{0}^{2} \delta_{p q}}{\lambda_{p}} \\
& +\frac{3 C \delta_{p q}}{\gamma^{2} \Gamma^{2}(3-\alpha)} \int_{0}^{t} \mathrm{~d} \tau \int_{0}^{t} \mathrm{~d} \tau^{\prime} e^{-\left|\tau-\tau^{\prime}\right| / \tau_{A}} \Xi_{\alpha}^{(p)}(\tau) \Xi_{\alpha}^{(q)}\left(\tau^{\prime}\right)
\end{aligned}
$$

with

$$
\Xi_{\alpha}^{(p)}(\tau)=\tau^{\alpha-1} E_{\alpha, \alpha}\left(-\left(\frac{\tau}{\tau_{0}}\right)^{\alpha} \lambda_{p}\right)
$$

In eqn (11)-(14) $E_{\alpha, \beta}(z)$ describes the Mittag-Leffler function 44

$$
E_{\alpha, \beta}(z)=\sum_{k=0}^{\infty} \frac{z^{k}}{\Gamma(\alpha k+\beta)}
$$

$\Gamma\left(\alpha ; x_{1}, x_{2}\right)=\int_{x_{1}}^{x_{2}} \mathrm{~d} x e^{-x} x^{\alpha-1}$ is the difference between two incomplete gamma functions, $\tau_{0}=\left(\frac{\gamma \Gamma(3-\alpha)}{k}\right)^{1 / \alpha}$, and $\lambda_{p}$ are the nonvanishing eigenvalues of matrix $\mathbf{A}$.

Equations (11)-13) allow to study different dynamical properties of the macromolecules. Here we concentrate on the monomeric displacement,

$$
\Delta \vec{R}_{n}(t)=\vec{R}_{n}(t)-\vec{R}_{n}(0)
$$

by considering its variance

$$
\sigma_{n}^{2}(t)=\left\langle\Delta \vec{R}_{n}(t)^{2}\right\rangle-\left\langle\Delta \vec{R}_{n}(t)\right\rangle^{2}=\left\langle\Delta \vec{R}_{n}(t)^{2}\right\rangle .
$$

Having a random choice of the bead, the mean-square displacement averaged over the structure reads

$$
\begin{aligned}
\sigma^{2}(t) & =\frac{1}{N} \sum_{n=1}^{N} \sigma_{n}^{2}(t)=\frac{1}{N}\left\langle\left(\vec{X}_{0}(t)-\vec{X}_{0}(0)\right)^{2}\right\rangle \\
& +\frac{1}{N} \sum_{p=1}^{N-1}\left(\left\langle\vec{X}_{p}^{2}(t)\right\rangle-2\left\langle\vec{X}_{p}(t) \vec{X}_{p}(0)\right\rangle+\left\langle\vec{X}_{p}^{2}(0)\right\rangle\right) \\
& =\sigma_{\mathrm{CM}, \mathrm{v}}^{2}(t)+\sigma_{\mathrm{CM}, \mathrm{a}}^{2}(t)+\sigma_{\mathrm{mon}, \mathrm{v}}^{2}(t)+\sigma_{\text {mon }, \mathrm{a}}^{2}(t)
\end{aligned}
$$

where

$$
\begin{gathered}
\sigma_{\mathrm{CM}, \mathrm{v}}^{2}(t)=\frac{2 l_{0}^{2}}{N \alpha \Gamma(\alpha)}\left(\frac{t}{\tau_{0}}\right)^{\alpha}, \\
\sigma_{\mathrm{CM}, \mathrm{a}}^{2}(t)=\frac{6 C \tau_{A}^{2 \alpha}}{N \gamma^{2} G_{\alpha}^{2}} \int_{0}^{t / \tau_{A}} \mathrm{~d} y e^{y} y^{\alpha-1} \Gamma\left(\alpha ; y, t / \tau_{A}\right),
\end{gathered}
$$

$$
\sigma_{\mathrm{mon}, \mathrm{v}}^{2}(t)=\frac{2 l_{0}^{2}}{N}\left(\frac{t}{\tau_{0}}\right)^{\alpha} \sum_{p=1}^{N-1} E_{\alpha, \alpha+1}\left(-\left(\frac{t}{\tau_{0}}\right)^{\alpha} \lambda_{p}\right),
$$

and

$\sigma_{\text {mon, }, \mathrm{a}}^{2}(t)=\frac{3 C}{N \gamma^{2} \Gamma^{2}(3-\alpha)} \sum_{p=1}^{N-1} \int_{0}^{t} \mathrm{~d} \tau \int_{0}^{t} \mathrm{~d} \tau^{\prime} e^{-\left|\tau-\tau^{\prime}\right| / \tau_{A}} \Xi_{\alpha}^{(p)}(\tau) \Xi_{\alpha}^{(p)}\left(\tau^{\prime}\right)$.

In order to control the evolution of the macromolecular size we are looking at the average value of the gyration radius at time $t, \underline{45}$

$$
\left\langle R_{g}^{2}(t)\right\rangle=\left\langle\frac{1}{N} \sum_{n=1}^{N}\left(\vec{R}_{n}(t)-\vec{R}_{c m}(t)\right)^{2}\right\rangle=\frac{1}{N} \sum_{p=1}^{N-1}\left\langle\vec{X}_{p}^{2}(t)\right\rangle,
$$

where $\vec{R}_{c m}(t)$ is the vector of the center of mass. Inserting eqn (13) into eqn (23) it follows readily

$$
\left\langle R_{g}^{2}(t)\right\rangle=\frac{l_{0}^{2}}{N} \sum_{p=1}^{N-1} \frac{1}{\lambda_{p}}+\sigma_{\text {mon,a }}^{2}(t) .
$$

The first term of eqn (24) describes the gyration radius of a macromolecule at thermal equilibrium ${ }^{17 / 45}$ and is independent of time. The second term, which has exactly the shape of eqn (22), grows with time, expressing the dynamics of swelling of the macromolecule due to the external active forces.

\subsection{Structures}

In this subsection we present different structures, whose dynamics in a viscoelastic and active bath we study in Sec 3. The connectivity of the networks leads to the spectrum $\left\{\lambda_{p}\right\}$ which can be characterized by the density of states $\rho(\lambda) \sim \lambda \frac{d_{s}}{2}-1$ and the related spectral dimension $d_{s}$. In other words, for eigenvalues $\left\{\lambda_{p}\right\}$ numbered in an ascending order the spectral dimension $d_{s}$ is reflected through a $\lambda_{p} \sim(p / N)^{2 / d_{s}}$ behavior. (We remark, however, that there are structures which possess some eigenvalues that do not follow the general tendency, vide infra.) Figure 2 shows the four different networks on which we focus in this paper, while figure 3 shows their eigenvalue spectra. One can readily see that the 


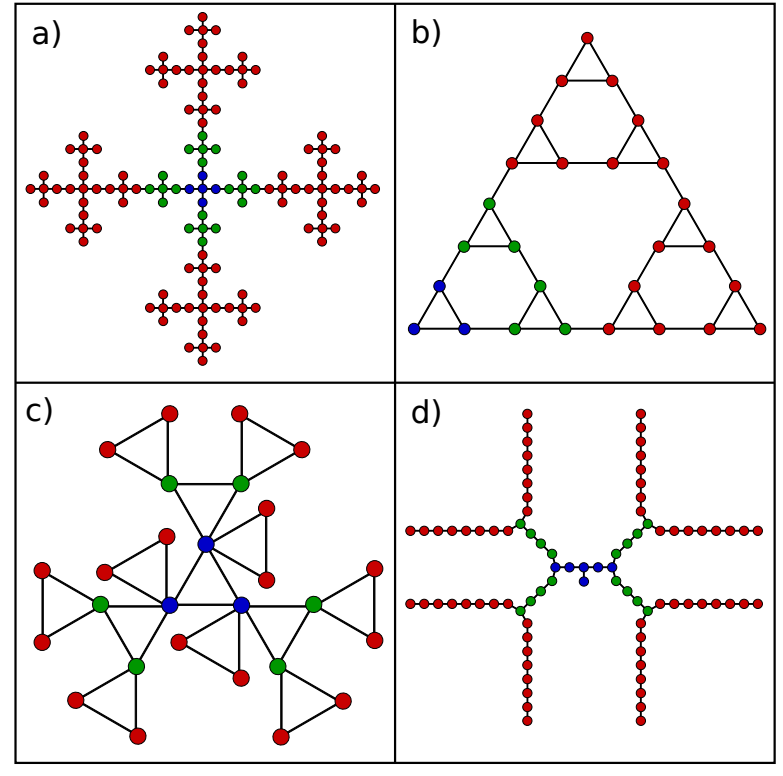

Fig. 2 Sketch of the structures studied in the paper: a) Vicsek fractal (VF) of functionality $f=4$. b) Dual Sierpiński gasket (DSG). c) Smallworld network (SWN) for parameter $d=3$. d) $N T_{D}$ tree characterized by parameter $k=2$. All structures are exemplified at generation $g=3$. The sketch is aimed to show only the connectivity of the structures; their spatial conformations in a three-dimensional space may come in vastly different forms.

Table 1 Parameters of the structures studied in the paper

\begin{tabular}{lll}
\hline Structure & Number of beads $N$ & $\begin{array}{l}\text { Number of distinct } \\
\text { eigenvalues } \lambda_{i}\end{array}$ \\
\hline VF, $f=3, g=7$ & 16384 & 2187 \\
VF, $f=4, g=7$ & 78125 & 2187 \\
VF, $f=6, g=7$ & 823543 & 2187 \\
DSG, $g=10$ & 59049 & 1535 \\
SWN $, d=3, g=12$ & 531441 & 4096 \\
NT $_{\mathrm{D}}, k=2, g=7$ & 21846 & 1761
\end{tabular}

spectra have degenerate eigenvalues related to the symmetry of the structures. This fact drastically reduces the computational efforts. Table 1 summarizes the molecular masses and the number of distinct eigenvalues of the structures studied in the paper.

\subsubsection{Vicsek fractals}

Vicsek fractals (VFs) are regular, symmetric structures that are constructed iteratively. 46 They are defined by two parameters, $f$ and $g$, where the functionality $f$ gives the number of neighbors of a branching point and the generation $g$ gives the number of iteration steps. Starting with a single bead, a VF of generation $g+1$ is constructed from $f+1$ VFs of generation $g$. With this, a VF of generation $g$ has $N=(f+1)^{g}$ beads. A VF of functionality $f=4$ and generation $g=3$ is shown in figure 2 .

For VFs the eigenvalues of matrix A can be calculated by solving iteratively the polynomial equation $25[47$

$$
\lambda^{(g+1)}\left(\lambda^{(g+1)}-3\right)\left(\lambda^{(g+1)}-f-1\right)=\lambda_{i}^{(g)},
$$

where $\lambda_{i}^{(g)} \neq 0$ is an eigenvalue of the fractal of generation $g$.
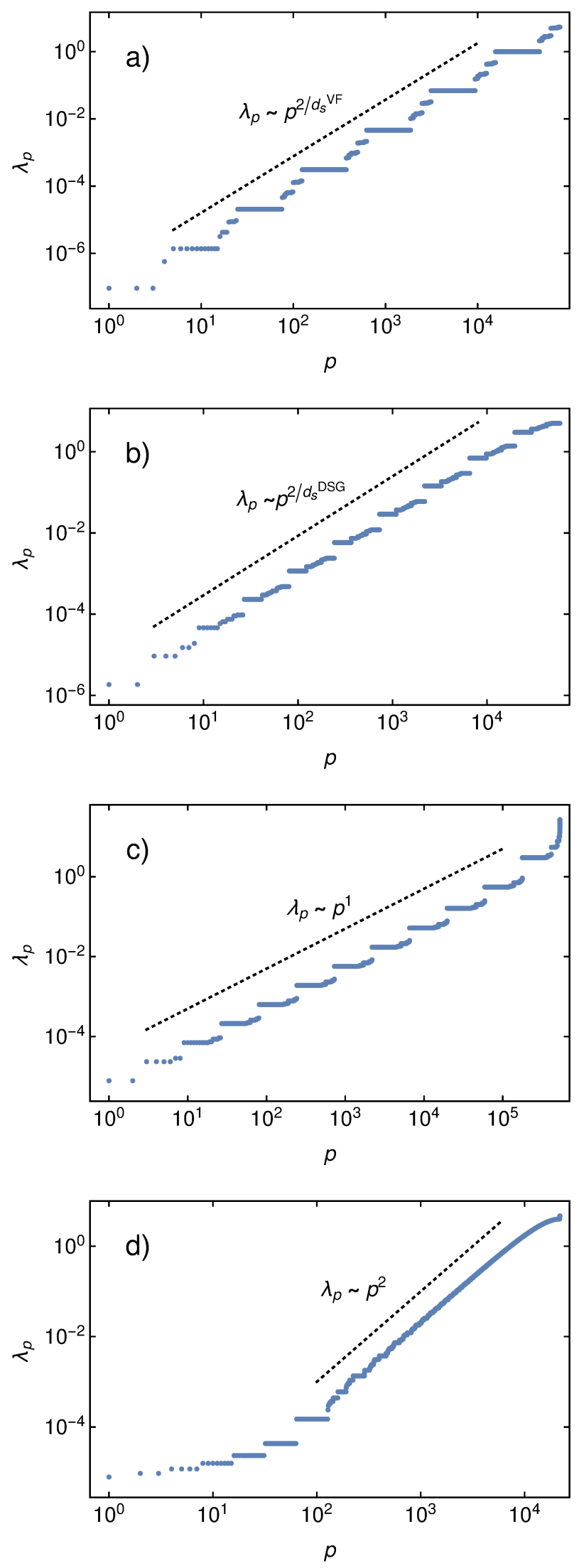

Fig. 3 Eigenvalue spectra of the Laplacian matrices for different macromolecular structures: a) VF of functionality $f=4$ and generation $g=7$. b) DSG of generation $g=10$. c) SWN with $d=3$ and generation $g=12$. d) $N T_{D}$ tree with $k=2$ and generation $g=7$ 
Eqn (25) yields three new eigenvalues for each eigenvalue of the previous generation. The ensuing set is supplemented at generation $g$ by the eigenvalues 1 with degeneracy $(f-2)(f+1)^{g-1}+1$, $f+1$ with degeneracy 1 , and 0 with degeneracy 1.25 Based on eqn (25) one can readily find the behavior of low eigenvalues, 2548 leading to the respective spectral dimension $d_{s}^{\mathrm{VF}}=$ $2 \ln (f+1) / \ln (3 f+3) \cdot 25$ As can be readily seen, a huge advantage of VFs is that their spectral dimension can be easily modified by using different values of functionality $f$. The eigenvalue spectrum of VFs is exemplified for functionality $f=4$ and generation $g=7$ in figure 3 a.

\subsubsection{Dual Sierpiński gaskets}

Dual Sierpiński gaskets (DSGs) are fractal structures with loops. 49 They can be constructed iteratively starting from a triangle of connected beads which represents the gasket of generation $g=1$. To construct the next generation, one takes three gaskets and connects them to a new triangle. Therefore, a DSG of generation $g$ consists of $N=3^{g}$ beads. A DSG of generation $g=3$ is shown in figure 2 p.

For DSGs, the eigenvalue spectrum can be calculated based on the iterative expression $24 \sqrt[50]{ }$

$$
\lambda_{g}^{(i) \pm}=\frac{5 \pm \sqrt{25-4 \lambda_{g-1}^{(i)}}}{2}
$$

which yields two new eigenvalues $\lambda_{g}^{(i) \pm}$ for each non-vanishing eigenvalue $\lambda_{g-1}^{(i)}$ of the previous generation. The eigenvalues 3 with degeneracy $\left(3^{g-1}+3\right) / 2$, 5 with degeneracy $\left(3^{g-1}-1\right) / 2$ and 0 with degeneracy 1 complete the spectrum of a DSG of generation $g$. The DSG spectrum is characterized by spectral dimension $d_{s}^{\text {DSG }}=2 \ln 3 / \ln 5.24$ An exemplary eigenvalue spectrum of a DSG of generation $g=10$ is displayed in figure 3 p.

\subsubsection{Small-world networks}

We turn to discuss a family of small-world networks (SWNs) that are defined by their generation $g$ and a parameter $d$, see ref. 33 . Starting with a complete graph of $d$ beads which represents an SWN of generation $g=1$, one constructs the next generation by replacing each bead with another complete graph. With that, the number of beads of an SWN of generation $g$ equals $N=d^{g}$, which in the case of $d=3$ coincides with the number of beads of the DSG. A SWN with $d=3$ and generation $g=3$ is shown in figure 2 .

The eigenvalues of SWNs can be calculated iteratively using 33

$$
\lambda_{g}^{(i) \pm}=\frac{1}{2}\left(d+\lambda_{g-1}^{(i)} \pm \sqrt{d^{2}+2 d \lambda_{g-1}^{(i)}+\left(\lambda_{g-1}^{(i)}\right)^{2}-4 \lambda_{g-1}^{(i)}}\right) .
$$

This equation yields two new eigenvalues $\lambda_{g}^{(i) \pm}$ for each eigenvalue $\lambda_{g-1}^{(i)}$ of the previous generation. The eigenvalue spectrum of an SWN of generation $g$ is completed by additional $d^{g}-2 d^{g-1}$ eigenvalues $d$. Considering eqn (27) for small eigenvalues leads to the spectral $d_{s}=2$, independently of the parameter $d . \frac{33}{33}$ This implies that the eigenvalue spectrum scales with $p, \lambda_{p} \sim p^{1}$, that can be observed in figure 3 for an SWN of parameter $d=3$ and

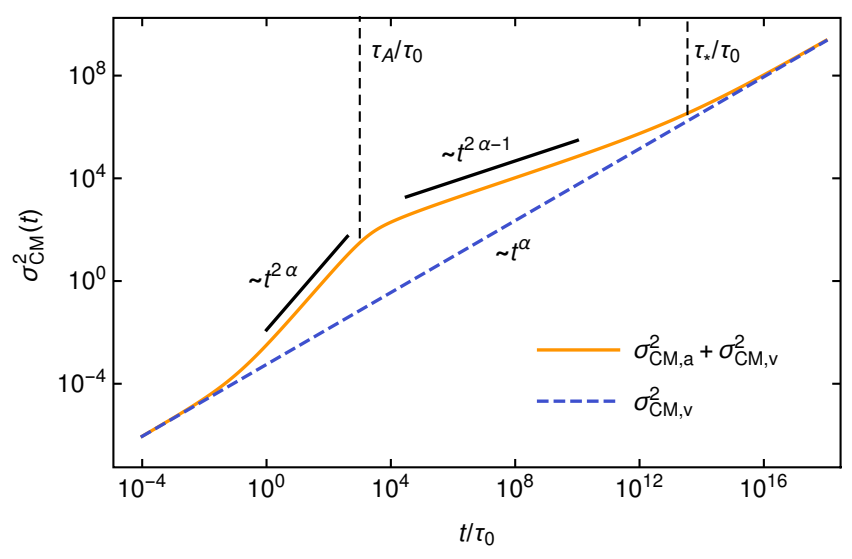

Fig. $4 \mathrm{MSD}$ of the center of mass of a macromolecule consisting of $N=10^{4}$ beads for $\alpha=0.7, l_{0}^{2}=3 / \Gamma(3-\alpha) \approx 2.57, \gamma=1, C=10$ and $\tau_{A}=10^{3} \tau_{0}$. The solid orange line shows the full MSD, whereas for the dashed blue line the active forces are turned off.

generation $g=12$.

\subsection{4 $N T_{D}$ trees}

An $N T_{D}$ tree is a treelike graph that is characterized by its generation $g$ and parameter $k \cdot \frac{30}{0}$ To get a tree of generation $g$ from generation $g-1$ one adds $k$ linear chains constisting of $2^{g}$ beads to each peripheral bead. Thus, the number of beads at generation $g$ is given by $N=1+\left(4^{g+1}-1\right) / 3$. An $N T_{D}$ tree with $k=2$ and generation $g=3$ is depicted in figure $2 \mathrm{~d}$.

The eigenvalue spectrum of the $N T_{D}$ trees is nonhomogenous. $30 \mid 32$ It is dominated by the behavior of the linear chains characterized by the spectral dimension $d_{s}=1$. However, the smallest eigenvalues that describe large relaxation times (which correspond to the relaxation of the branches and subbranches as a whole $\frac{51+53}{53}$ and related to large-scale characteristics such as gyration radius at the thermal equilibrium ${ }^{45}$ ) march to a different tune. Though the smallest eigenvalues represent a discrete set (at the thermodynamic limit they build a set of the Lebesgue measure zero), they possess a $\lambda_{p} \sim(p / N)^{2 \ln 2 / \ln (2 k)}$ behavior. ${ }^{30}$ An exemplary spectrum for an $N T_{D}$ tree of generation $g=7$ and with parameter $k=2$ is shown in figure 3 .

\section{Results}

\subsection{Analysis of $\sigma^{2}(t)$}

We start our analysis of the mean squared displacement (MSD) $\sigma^{2}(t)$ by looking first on the motion of the center of mass, represented by the terms $\sigma_{\mathrm{CM}, \mathrm{v}}^{2}(t)+\sigma_{\mathrm{CM}, \mathrm{a}}^{2}(t)$ of eqn $(18)$. We notice that these terms include only the number of beads, but no information about the topology of the macromolecule. This means that the center of mass moves analogously for all macromolecules of the same molecular mass. The term $\sigma_{\mathrm{CM}, \mathrm{v}}^{2}(t)$ represents the (subdiffusive) motion of the center of mass in a viscoelastic bath without active forces, whereas $\sigma_{\mathrm{CM}, \mathrm{a}}^{2}(t)$ is added when active forces come into play. In figure 4 we show the MSD of the center of mass in a viscoelastic environment, with and without active forces.

As can be inferred from eqn (19) and shown in figure 4, in the passive case the viscoelastic bath leads to a subdiffusive $t^{\alpha}$. 
behavior of the center of mass. The active forces described by $\sigma_{\mathrm{CM}, \mathrm{a}}^{2}(t)$ enrich for $\tau_{0} \ll t \ll \tau_{*}$ (see the definition of $\tau_{*}$ in eqn (31) below) the dynamics of the center of mass. For short times $t \ll \tau_{A}$ one can use in eqn 20 that $\exp \left(-\left|\tau-\tau^{\prime}\right| / \tau_{A}\right) \approx 1$ leads to

$$
\frac{3 C}{N \gamma^{2} G_{\alpha}^{2}} \int_{0}^{t} \mathrm{~d} \tau \int_{0}^{t} \mathrm{~d} \tau^{\prime} \tau^{\alpha-1}\left(\tau^{\prime}\right)^{\alpha-1}=\left(\frac{3 C}{N \gamma^{2} \alpha^{2} G_{\alpha}^{2}}\right) t^{2 \alpha} .
$$

Thus active forces cause for $\alpha>1 / 2$ a superdiffusive behavior. On the other hand, for long times $t \gg \tau_{A}$ the function $\Gamma\left(\alpha ; y, t / \tau_{A}\right)$ inside the integral of eqn (20) can be well approximated through the incomplete Gamma function, $\Gamma\left(\alpha ; y, t / \tau_{A}\right) \approx \Gamma(\alpha ; y)$. Therefore, the integral of eqn (20) can be split into two parts

$$
\int_{0}^{t / \tau_{A}} \mathrm{~d} y e^{y} y^{\alpha-1} \Gamma(\alpha ; y) \simeq I_{\alpha}(x)+\frac{1}{2 \alpha-1}\left(\frac{t}{\tau_{A}}\right)^{2 \alpha-1}
$$

with $I_{\alpha}(x)=\int_{0}^{x} \mathrm{~d} y e^{y} y^{\alpha-1} \Gamma(\alpha ; y)-x^{2 \alpha-1} /(2 \alpha-1)$, where $x \gg 1$. With this the long time behavior of $\sigma_{\mathrm{CM}, \mathrm{a}}^{2}(t)$ is dominated by

$$
\left(\frac{6 C \tau_{A}}{(2 \alpha-1) N \gamma^{2} G_{\alpha}^{2}}\right) t^{2 \alpha-1}
$$

Comparing the above eqn (30) with $\sigma_{\mathrm{CM}, \mathrm{v}}^{2}(t)$ of eqn 19) one finds (for $\alpha<1$ ) the time $\tau_{*}$ at which the activity becomes irrelevant,

$$
\tau_{*}=\left(\frac{l_{0}^{2}(2 \alpha-1) \gamma^{2} \Gamma(\alpha) \Gamma^{2}(3-\alpha)}{3 \tau_{0}^{\alpha} \alpha C \tau_{A}}\right)^{1 /(\alpha-1)} .
$$

Summarizing, the MSD of the center of mass displays the $t^{\alpha}$ behavior for $t \ll \tau_{0}$ and $t \gg \tau_{*}$ due to the viscoelastic bath; for the in-between times one has then $t^{2 \alpha}$ and $t^{2 \alpha-1}$ behaviors for $\tau_{0} \ll t \ll \tau_{A}$ and $\tau_{A} \ll t \ll \tau_{*}$, respectively, originating from the active forces. For $1 / 2<\alpha<1$ the latter two regimes show a change from a superdiffusive to subdiffusive behavior, that has been also observed for single particles in active viscoelastic environments. $14 \mid 54$

We turn to the analysis of the other two terms of $\sigma^{2}(t)$, $\sigma_{\text {mon,v }}^{2}(t)+\sigma_{\text {mon,a }}^{2}(t)$, which depend on the particular architecture of the network. The term $\sigma_{\text {mon,v }}^{2}(t)$ is responsible for the monomer motion in a passive viscoelastic bath. In order to analyze its behavior we use that the density of the eigenvalues $\rho(\lambda)$ scales as $\lambda^{d_{s} / 2-1}$ and make a continuous transition from the sum to an integral, i.e.,

$$
\begin{aligned}
& t^{\alpha} \sum_{p=1}^{N-1} E_{\alpha, \alpha+1}\left(-\left(\frac{t}{\tau_{0}}\right)^{\alpha} \lambda_{p}\right) \rightarrow \\
& t^{\alpha\left(1-d_{s} / 2\right)} \tau_{0}^{\alpha d_{s} / 2} \int_{\lambda_{\min } \cdot\left(t / \tau_{0}\right)^{\alpha}}^{\lambda_{\max } \cdot\left(t / \tau_{0}\right)^{\alpha}} \mathrm{d} x x^{d_{s} / 2-1} E_{\alpha, \alpha+1}(-x),
\end{aligned}
$$

where $\lambda_{\min }$ is the smallest non-zero eigenvalue and $\lambda_{\max }$ is the biggest eigenvalue of the Laplacian matrix $\mathbf{A}$. For small values $x \ll 1$, the Mittag-Leffler function $E_{\alpha, \alpha+1}(-x)$ can be approximated by the constant $1 / \Gamma(\alpha+1)$. Under this approximation the integral can be readily computed, yielding a time dependence proportional to $t^{\alpha}$. This time regime holds for $t \ll \tau_{0} / \lambda_{\max }^{1 / \alpha}$. For large values $x \gg 1$, the Mittag-Leffler function $E_{\alpha, \alpha+1}(-x)$ behaves as $1 / x$. The integral then yields a time dependence propor- tional to $t^{0}$, i.e, a constant behavior. This time regime holds for times $t \gg \tau_{0} / \lambda_{\min }^{1 / \alpha}$. For intermediate times $\tau_{0} / \lambda_{\max }^{1 / \alpha} \ll t \ll \tau_{0} / \lambda_{\min }^{1 / \alpha}$ we divide the integral of eqn (32) into three parts and use the small and long time behaviors of the Mittag-Leffler function discussed above,

$$
\begin{gathered}
t^{\alpha\left(1-d_{s} / 2\right)} \tau_{0}^{\alpha d_{s} / 2} \int_{\lambda_{\min } \cdot\left(t / \tau_{0}\right)^{\alpha}}^{\lambda_{\max } \cdot\left(t / \tau_{0}\right)^{\alpha}} \mathrm{d} x x^{d_{s} / 2-1} E_{\alpha, \alpha+1}(-x) \\
\approx t^{\alpha\left(1-d_{s} / 2\right)} \tau_{0}^{\alpha d_{s} / 2}\left[\frac{2}{d_{s} \Gamma(\alpha+1)}\left(g_{1}^{d_{s} / 2}-\left(\frac{\lambda_{\min } t^{\alpha}}{\tau_{0}^{\alpha}}\right)^{d_{s} / 2}\right)\right. \\
\left.\quad+c_{1}\left(d_{s}\right)+\frac{1}{\frac{d_{s}}{2}-1}\left(\left(\frac{\lambda_{\max } t^{\alpha}}{\tau_{0}^{\alpha}}\right)^{d_{s} / 2-1}-g_{2}^{d_{s} / 2-1}\right)\right]
\end{gathered}
$$

where $g_{1} \ll 1$ and $g_{2} \gg 1$ are the limits of the approximations for the $E_{\alpha, \alpha+1}(-x)$.

Now, for spectral dimensions $d_{s}$ close to 1 and intermediate times $\tau_{0} / \lambda_{\max }^{1 / \alpha} \ll t \ll \tau_{0} / \lambda_{\min }^{1 / \alpha}$ the constant $c_{1}\left(d_{s}\right)=$ $\int_{g_{1}}^{g_{2}} \mathrm{~d} x x^{d s / 2-1} E_{\alpha, \alpha+1}(-x)$ is the dominating term of eqn 33 . This means the MSD is proportional to $t^{\alpha\left(1-d_{s} / 2\right)}$. The range of validity of this power law depends on $d_{s}$. So, for $d_{s}$ advancing to 2, the last term of eqn (33) is getting more important and eventually diverges, which indicates a collapse of the $t^{\alpha\left(1-d_{s} / 2\right)}$ time dependence. Additionally, the exponent $\alpha\left(1-d_{s} / 2\right)$ is getting smaller with increasing spectral dimension and finally vanishes. Therefore, we take a closer look at the limiting case $d_{s}=2$. Using that the spectral density for $d_{s}=2$ is constant we obtain

$$
\begin{aligned}
& \tau_{0}^{\alpha} \int_{\lambda_{\min } \cdot\left(t / \tau_{0}\right)^{\alpha}}^{\lambda_{\max } \cdot\left(t / \tau_{0}\right)^{\alpha}} \mathrm{d} x E_{\alpha, \alpha+1}(-x) \\
\approx & \tau_{0}^{\alpha}\left[\frac{1}{\Gamma(\alpha+1)}\left(g_{1}-\frac{\lambda_{\min } t^{\alpha}}{\tau_{0}^{\alpha}}\right)+c_{1}\left(d_{s}=2\right)+\ln \left(\frac{\lambda_{\max } t^{\alpha}}{\tau_{0}^{\alpha} g_{2}}\right)\right] .
\end{aligned}
$$

Since the first term is getting suppressed by $\lambda_{\min }$ we get for $d_{s}=2$ a logarithmic dependence of $\sigma_{\text {mon,v }}^{2}(t)$ in time over a large time scale.

Finally we consider the fourth term of eqn $(18), \sigma_{\text {mon,a }}^{2}(t)$, related to the monomer motion under active forces. First we look at times $t \ll \tau_{A}$, for which one has $\exp \left(-\left|\tau-\tau^{\prime}\right| / \tau_{A}\right) \approx 1$. We then get

$$
\sigma_{\text {mon, }, \mathrm{a}}^{2}(t) \approx \frac{3 C}{N \gamma^{2} \Gamma^{2}(3-\alpha)} t^{2 \alpha} \sum_{p=1}^{N-1} E_{\alpha, \alpha+1}^{2}\left(-\frac{t^{\alpha}}{\tau_{0}^{\alpha}} \lambda_{p}\right) .
$$

Following the calculation of eqn (32) ff., we make a transition from the sum in eqn (35) to an integral,

$$
\begin{aligned}
& t^{2 \alpha} \sum_{p=1}^{N-1} E_{\alpha, \alpha+1}^{2}\left(-\frac{t^{\alpha}}{\tau_{0}^{\alpha}} \lambda_{p}\right) \rightarrow \\
& t^{\alpha\left(2-d_{s} / 2\right)} \tau_{0}^{\alpha d_{s} / 2} \int_{\lambda_{\min } \cdot\left(t / \tau_{0}\right)^{\alpha}}^{\lambda_{\max } \cdot\left(t / \tau_{0}\right)^{\alpha}} \mathrm{d} x x^{d_{s} / 2-1} E_{\alpha, \alpha+1}^{2}(-x),
\end{aligned}
$$

For times $t \ll \tau_{0} / \lambda_{\max }^{1 / \alpha}$, we again approximate the Mittag-Leffler function $E_{\alpha, \alpha+1}(-x)$ by $1 / \Gamma(\alpha+1)$. Performing the integration 
yields a time dependence proportional to $t^{2 \alpha}$. Now, we split the integral into

$$
\begin{aligned}
& t^{\alpha\left(2-d_{s} / 2\right)} \tau_{0}^{\alpha d_{s} / 2}\left[\frac{2}{d_{s} \Gamma^{2}(\alpha+1)}\left(g_{1}^{d_{s} / 2}-\left(\frac{\lambda_{\min } t^{\alpha}}{\tau_{0}^{\alpha}}\right)^{d_{s} / 2}\right)\right. \\
& \left.+c_{2}\left(d_{s}\right)+\frac{1}{\frac{d_{s}}{2}-2}\left(\left(\frac{\lambda_{\max } t^{\alpha}}{\tau_{0}^{\alpha}}\right)^{d_{s} / 2-2}-g_{2}^{d_{s} / 2-2}\right)\right]
\end{aligned}
$$

with $c_{2}\left(d_{s}\right)=\int_{g_{1}}^{g_{2}} \mathrm{~d} x x^{d s / 2-1} E_{\alpha, \alpha+1}^{2}(-x)$. At intermediate times $\tau_{0} / \lambda_{\max }^{1 / \alpha} \ll t \ll \tau_{A}$ the second term yields a

$$
\sigma_{\mathrm{mon}, \mathrm{a}}^{2}(t) \sim t^{\alpha\left(2-d_{s} / 2\right)}
$$

power law.

When $t$ approaches the time $\tau_{A}$ the exponential function $\exp \left(-\left|\tau-\tau^{\prime}\right| / \tau_{A}\right)$ starts to decay and the behavior $t^{\alpha\left(2-d_{s} / 2\right)}$ breaks down. Further, for $t \gg \tau_{A}$ the active noise acts as a white noise (but of a higher temperature than the initial one), i.e., we use $\exp \left(-\left|\tau-\tau^{\prime}\right| / \tau_{A}\right) \approx 2 \tau_{A} \delta\left(\tau-\tau^{\prime}\right)$. We then get

$$
\begin{aligned}
\sigma_{\text {mon,a }}^{2}(t) & \simeq \sigma_{\text {mon,a }}^{2}\left(\tau_{A}\right) \\
& +\frac{6 C \tau_{A}}{N \gamma^{2} \Gamma^{2}(3-\alpha)} \sum_{p=1}^{N-1} \int_{\tau_{A}}^{t} \mathrm{~d} \tau \tau^{2 \alpha-2} E_{\alpha, \alpha}^{2}\left(-\frac{\tau^{\alpha}}{\tau_{0}^{\alpha}} \lambda_{p}\right)
\end{aligned}
$$

To analyze the behavior of the second term of eqn (39) we make a continuous transition and look at the integral

$$
\tau_{0}^{\alpha d_{s} / 2} \int_{\tau_{A}}^{t} \mathrm{~d} \tau \tau^{\alpha\left(2-d_{s} / 2\right)-2} \int_{\lambda_{\min } \cdot\left(\tau / \tau_{0}\right)^{\alpha}}^{\lambda_{\max } \cdot\left(\tau / \tau_{0}\right)^{\alpha}} \mathrm{d} x x^{d_{s} / 2-1} E_{\alpha, \alpha}^{2}(-x) .
$$

For times $\tau_{A} \ll t \ll \tau_{0} / \lambda_{\text {min }}^{1 / \alpha}$ the second integral in eqn (40) can be approximated by a constant leading (for $d_{s} \neq 4-2 / \alpha$ ) to a result that is proportional to $\left(t^{\alpha\left(2-d_{s} / 2\right)-1}-\tau_{A}^{\alpha\left(2-d_{s} / 2\right)-1}\right) /\left[\alpha\left(2-d_{s} / 2\right)-1\right]$. Depending on $d_{s}$ and $\alpha$, we then get the following behavior

$$
\sigma_{\text {mon }, \mathrm{a}}^{2}(t) \sim \begin{cases}t^{\alpha\left(2-d_{s} / 2\right)-1}-\mathrm{const}, & \text { for } d_{s}<4-\frac{2}{\alpha} \\ \log (t), & \text { for } d_{s}=4-\frac{2}{\alpha} \\ \operatorname{const}-t^{\alpha\left(2-d_{s} / 2\right)-1}, & \text { for } d_{s}>4-\frac{2}{\alpha}\end{cases}
$$

Finally, for times $t \gg \tau_{0} / \lambda_{\min }^{1 / \alpha}, \sigma_{\text {mon,a }}^{2}(t)$ saturates to a constant value.

\subsection{Control of swelling under active forces}

Before going to the illustration of the findings of Section 3.1, we take a look at the gyration radius $\left\langle R_{g}^{2}(t)\right\rangle$, eqn (24), which consists from the equilibrium value of the gyration radius $\left\langle R_{g}^{2}(0)\right\rangle$ and from $\sigma_{\text {mon,a }}^{2}(t)$ (eqn (22), vide supra). The latter term describes a swelling of the macromolecule under active forces and it can eventually lead to its overstretching. Therefore, by looking at the change of the gyration radius, one can control the limits of parameters $C$ and $\tau_{A}$ characterizing strength and endurance of the active forces. Given that the equilibrium distances in Gaussian macromolecules have a square root dependence on the corresponding curvilinear path, we have decided for this study to limit

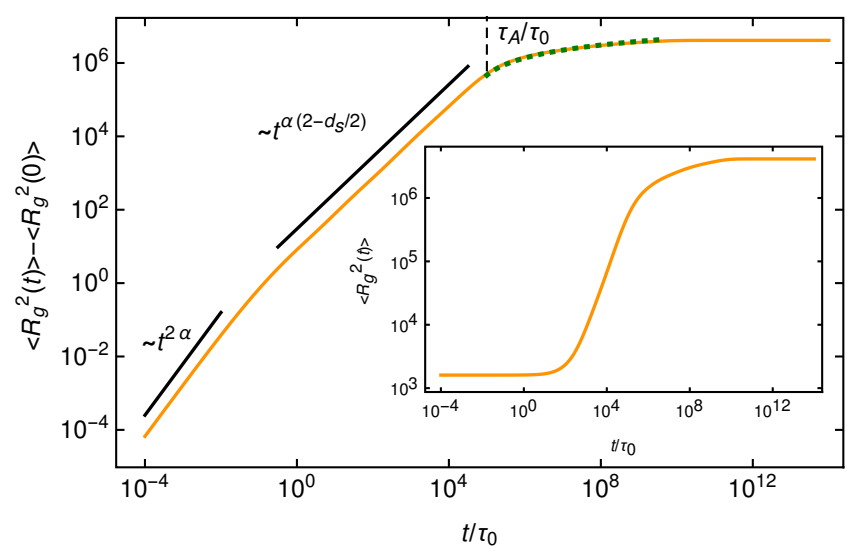

Fig. 5 Mean squared gyration radius for VF of generation $g=7$ and functionality $f=4$. The other parameters are $\alpha=0.7, l_{0}^{2}=3 / \Gamma(3-\alpha) \approx$ 2.57, $\gamma=1, C=10$ and $\tau_{A}=10^{5} \tau_{0}$. In the main figure the equilibrium size is subtracted while in the inset it is included.

the parameters $C$ and $\tau_{A}$ to the values that do not allow $\left\langle R_{g}^{2}(\infty)\right\rangle$ to exceed the order of $\left\langle R_{g}^{2}(0)\right\rangle^{2} / l_{0}^{2}$.

Figure 5 shows the gyration radius for a VF of generation $g=7$ and functionality $f=4$. Here we can see the discussed above time evolution of $\sigma_{\text {mon,a }}^{2}(t)=\left\langle R_{g}^{2}(t)\right\rangle-\left\langle R_{g}^{2}(0)\right\rangle$. This starts to grow according to a $t^{2 \alpha}$ power law, followed by the regime proportional to $t^{\alpha\left(2-d_{s} / 2\right)}$ (before reaching $\tau_{A}$ ) and then slowly saturates (following eqn (41), here $d_{s}>4-2 / \alpha$ ) to a constant value.

\subsection{Monomer dynamics of different networks}

In this subsection we make an explicit illustration of the MSD of the monomers belonging to various structures presented in Section 2.2. For all considered examples the parameters characterizing the active forces are chosen bearing in mind the discussion of Section 3.2. In particular, in all cases the correlation time $\tau_{A}$ of the active forces is about one percent of the large-scale characteristic time $\tau_{0} / \lambda_{\min }$ of the structures.

Figure 6 shows passive (dashed lines) and active (solid lines) dynamics of the networks' monomers. Independently of the networks' architecture and the bath activity, at very small and very large times one observes a universal $t^{\alpha}$ time behavior reflecting the viscoelasticity of the bath. The initial behavior $t^{2 \alpha}$ of the term $\sigma_{\text {mon,a }}^{2}(t)$ (eqn (22) ) is suppressed by the initial viscoelastic one $\left(t^{\alpha}\right)$ due to the modesty of the active forces.

Starting with times $t>\tau_{0}$ one encounters the differences between the active and passive case. For these times, in the passive viscoelastic bath the monomer motion is much slower than for the case when also the active forces are present. In the passive environment one observes a $t^{\alpha\left(1-d_{s} / 2\right)}$ behavior for $d_{s}<2$ and $\log (t)$ for $d_{s}=2$. In the active case one has a $t^{\alpha\left(2-d_{s} / 2\right)}$ power law, whose range for $d_{s}=2$ marginalizes (although one can certainly find the corresponding $t^{\alpha}$ power law in the active component $\sigma_{\text {mon,a }}^{2}(t)$ of monomer MSD, see black dotted line on Figure 6r). For $N T_{D}$ trees one clearly observes the scaling exponents $t^{\alpha / 2}$ in the passive case and $t^{3 \alpha / 2}$ in the active case, the same as for a linear chain ${ }^{4}$. Thus, the ground states related to the equilibrium size 

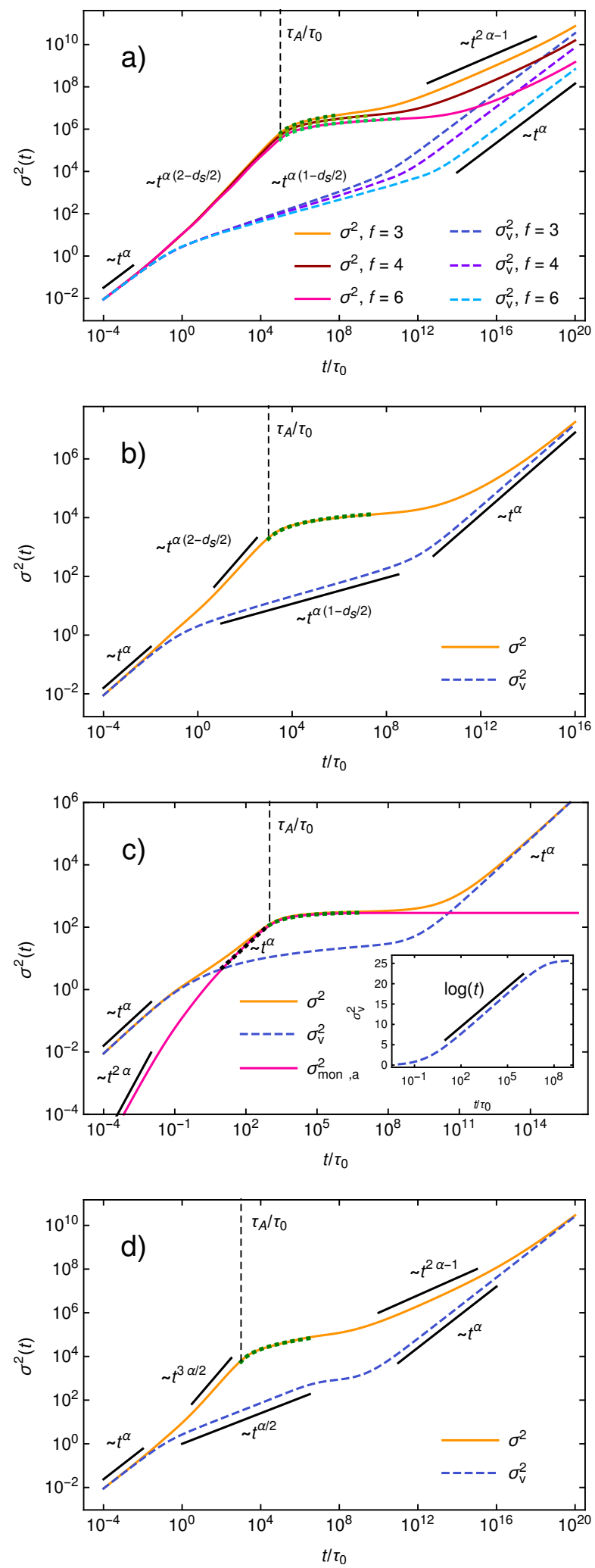

Fig. 6 Monomer MSD of different macromolecul|ar structures: ai) W/Fs off different functionalities arah geareationg $g=7.7 \mathrm{bb}$ ) D

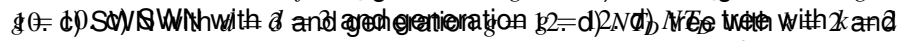

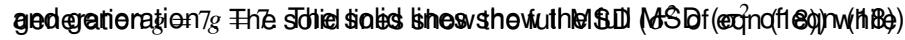

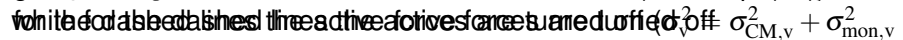

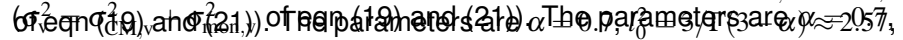

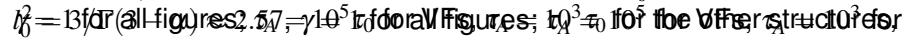

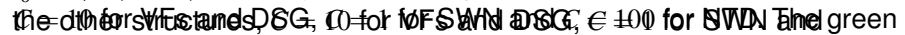
dotteddifos NeTere tant for the monomer dynamics (these states reveal themselves of the trees and hence to their fractal dimension are unimportant for the monomer dynamics (these states reveal themselves of mass motion,. In general, for times $\tau_{0}<t<\tau_{A}$ the structural only slightly in the passive case just before reaching the center of mass motion). In general, for times $\tau_{0}<t<\tau_{t}$ the structural more pronounced in the passive case, becauselittle changes in $d_{s}$ differences that are reflected in the spectral dimension $\left.d_{s}\right)$ are $d_{s}$ more pronounced un the passive case, because little changes in $d_{s}$ Moreover, the $t^{\alpha}\left(2-d_{s} / 2\right.$ power law of the active case holds only Moreover, the $t_{\text {an }}$ which tigh has to be much smaller than the maximal relaxation time of the structures in order apt to overstretch.them. rela oleederetehbatenchanges, following the behavior of eqn (41). As

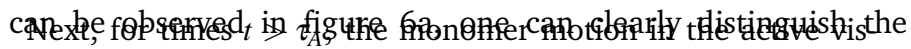

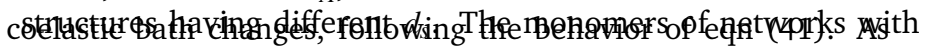

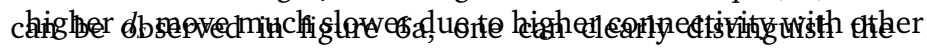

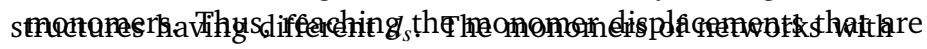

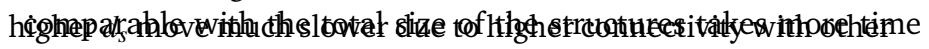
nfon

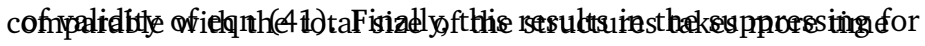

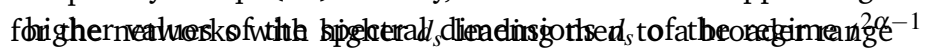

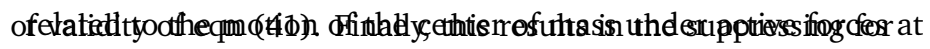
hïghes talues $\varangle$ of the spectral dimensions $d_{s}$ of the regime $t^{2 \alpha-1}$ related to the motion of the center of mass under active forces at thines Gonclusions

4n tormekusidans studied the dynamical behavior of macromolecular networks in an active and viscoelastic bath. In doing In this work we have studied the dynamical behavior of macroso we have analyzed the MSD of monomers, which can be formumolecular networks in an active and viscoelastic bath. In doing so, we have analyzed the MSD of monomers, which can be formuof the respective network. The active forces lead to a tremendous lated based on the ergenvalue spectrum of the Laplacian matrix change in the dynamics of the macromolecules. At the intermediate times one observes structure-dependent dynamics of swelling, change in the dynamics of the macromolecules. At the intermediwhich is characterized through the spectral dimension $d_{\text {pling }}$ The monomer motion in this regime is much faster than in the passive viscoelastic bath that can. be even superdiffusive for higher ex-
monomer motion in this regime is much paster than in the passive vocoents a characterizing the bath an lower spectral dipensions flurorescenterizing the connectivity of the structure. At it ines that can be higher than the the tyical duration of action of the active forces, the daynamigs of monemars shows down cheing eren slower than net the passive case reveflecting the relaxation of tensiop caysed by dthe active forces aftefe atcearlier times the dynamics of monomers

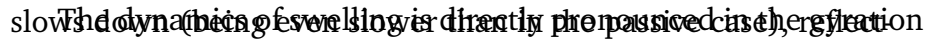

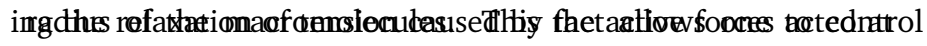

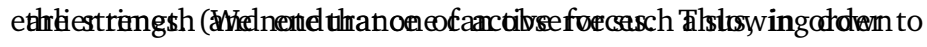

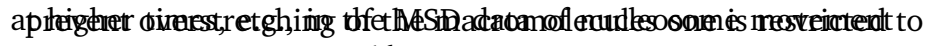

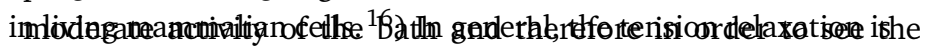

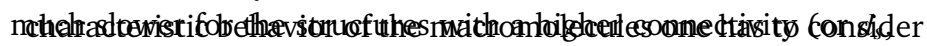

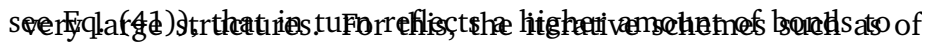

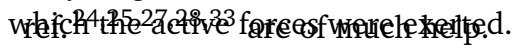

The dynamigs of swelling is directly pronounced in the toyra tion radius of the macromolequles Therefore if one kan define $a_{\text {n }}$

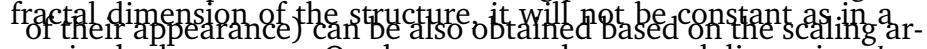
passive batto apymore on the contrafy the spectral dimension d is solely defined by the Laplacian matrix A so that for the structure with a quenched connectivity it is a constant. Monitoring the growth of a macromolecule allows one to control the strength and endurance of active forces. Thus, in order to prevent overstretch-
spectrum are necessary. 
ing of the macromolecules one is restricted to moderate activity of the bath and therefore in order to see the characteristic behavior of the macromolecules one has to consider very large structures. For this, the iterative schemes such as of ref. $\frac{24|25| 2728 \mid 33}{2}$ are of much help.

This study has aimed to bridge microscopic connectivity with the macroscopic behavior. The ensuing power laws (in the regions of their appearance) can be also obtained based on the scaling arguments, $\frac{12}{12}$ if one assumes self-similarity of the fractal networks. However, in case of the structures with irregular Laplacian spectra, such as $N T_{D}$ trees, and for a detailed picture of the full functional evolution rigorous calculations that account for the whole spectrum are necessary.

\section{Acknowledgments}

The authors appreciate the fruitful discussions with Tanja Schilling and Marcus Müller and acknowledge support by the state of Baden-Württemberg through bwHPC.

\section{Conflicts of interest}

There are no conflicts to declare.

\section{References}

1 S. C. Weber, A. J. Spakowitz and J. A. Theriot, Phys. Rev. Lett., 2010, 104, 238102.

2 S. C. Weber, A. J. Spakowitz and J. A. Theriot, Proc. Natl. Acad. Sci., 2012, 109, 7338-7343.

3 A. Ghosh and N. S. Gov, Biophys. J., 2014, 107, 1065-1073.

4 H. Vandebroek and C. Vanderzande, Phys. Rev. E, 2015, 92, 060601.

5 A. Kaiser, S. Babel, B. ten Hagen, C. von Ferber and H. Löwen, J. Chem. Phys., 2015, 142, 124905.

6 N. Samanta and R. Chakrabarti, J. Phys. A: Math. Theor, 2016, 49, 195601.

7 J. Shin, A. G. Cherstvy, W. K. Kim and R. Metzler, New J. Phys., 2015, 17, 113008.

8 T. Eisenstecken, G. Gompper and R. G. Winkler, Polymers, 2016, 8, 304.

9 T. Eisenstecken, G. Gompper and R. G. Winkler, J. Chem. Phys., 2017, 146, 154903.

10 D. Osmanovic and Y. Rabin, Soft Matter, 2017, 13, 963-968.

11 T. Saito and T. Sakaue, Phys. Rev. E, 2017, 95, 042143.

12 T. Sakaue and T. Saito, Soft Matter, 2017, 13, 81-87.

13 J. Shin, A. G. Cherstvy, W. K. Kim and V. Zaburdaev, Phys. Chem. Chem. Phys., 2017, 19, 18338-18347.

14 H. Vandebroek and C. Vanderzande, J. Stat. Phys., 2017, 167, 14-28.

15 H. Vandebroek and C. Vanderzande, Soft Matter, 2017, 13, 2181-2191.

16 S. Shinkai, T. Nozaki, K. Maeshima and Y. Togashi, PLOS Comput. Biol., 2016, 12, e1005136.

17 M. Doi and S. F. Edwards, The theory of polymer dynamics, Oxford university press, 1988.

18 E. Lieberman-Aiden et al., Science, 2009, 326, 289-293.
19 S. Reuveni, R. Granek and J. Klafter, Proc. Natl. Acad. Sci., 2010, 107, 13696-13700.

20 L. A. Mirny, Chromosome Res., 2011, 19, 37-51.

21 G. Fudenberg, G. Getz, M. Meyerson and L. A. Mirny, Nat. Biotechnol., 2011, 29, 1109-1113.

22 A. Y. Grosberg, Polym. Sci. Ser. C, 2012, 54, 1-10.

23 L. M. Almassalha et al., Sci. Rep., 2017, 7, 41061.

24 A. Blumen and A. Jurjiu, J. Chem. Phys., 2002, 116, 26362641.

25 A. Blumen, C. von Ferber, A. Jurjiu and T. Koslowski, Macromolecules, 2004, 37, 638-650.

26 M. Dolgushev, T. Guérin, A. Blumen, O. Bénichou and R. Voituriez, Phys. Rev. Lett., 2015, 115, 208301.

27 Z. Zhang, Y. Qi, S. Zhou, Y. Lin and J. Guan, Phys. Rev. E, 2009, 80, 016104.

28 A. Jurjiu, T. L. Biter and F. Turcu, J. Chem. Phys., 2017, 146, 034902.

29 E. Ben-Isaac, Y. Park, G. Popescu, F. L. Brown, N. S. Gov and Y. Shokef, Phys. Rev. Lett., 2011, 106, 238103.

30 R. Burioni and D. Cassi, Phys. Rev. E, 1994, 49, R1785.

31 R. Burioni and D. Cassi, Phys. Rev. E, 1995, 51, 2865.

32 O. Mülken, S. Heinzelmann and M. Dolgushev, J. Stat. Phys., 2017, 167, 1233-1243.

33 H. Liu, M. Dolgushev, Y. Qi and Z. Zhang, Sci. Rep., 2015, 5, 9024.

34 N. Biggs, Algebraic graph theory, Cambridge university press, 1993.

35 R. Zwanzig, Phys. Rev., 1961, 124, 983.

36 R. F. Fox, Phys. Rep., 1978, 48, 179-283.

37 E. Cortés, B. J. West and K. Lindenberg, J. Chem. Phys., 1985 , 82, 2708-2717.

38 W. Deng and E. Barkai, Phys. Rev. E, 2009, 79, 011112.

39 D. Panja, J. Stat. Mech., 2010, P06011.

40 R. Kubo, Rep. Prog. Phys., 1966, 29, 255.

41 I. M. Tolić-Nørrelykke, E.-L. Munteanu, G. Thon, L. Oddershede and K. Berg-Sørensen, Phys. Rev. Lett., 2004, 93, 078102.

42 N. Fakhri, A. D. Wessel, C. Willms, M. Pasquali, D. R. Klopfenstein, F. C. MacKintosh and C. F. Schmidt, Science, 2014, 344, 1031-1035.

$43 \mathrm{~K}$. Oldham and J. Spanier, The fractional calculus theory and applications of differentiation and integration to arbitrary order, Elsevier, 1974.

44 G. Mittag-Leffler, C. R. Acad. Sci., 1903, 137, 554-558.

45 J.-U. Sommer and A. Blumen, J. Phys. A: Math. Gen., 1995, 28, 6669 .

46 T. Vicsek, J. Phys. A: Math. Gen., 1983, 16, L647.

47 C. Jayanthi and S. Wu, Phys. Rev. B, 1993, 48, 10199.

48 M. Dolgushev, D. A. Markelov, F. Fürstenberg and T. Guérin, Phys. Rev. E, 2016, 94, 012502.

49 M. Sierpiński, C. R. Acad. Sci., 1915, 160, 302-305.

50 M. G. Cosenza and R. Kapral, Phys. Rev. A, 1992, 46, 1850.

51 C. Cai and Z. Y. Chen, Macromolecules, 1997, 30, 5104-5117. 
52 Y. Y. Gotlib and D. A. Markelov, Polym. Sci. Ser. A, 2002, 44, 1341-1350.

53 J. Grimm and M. Dolgushev, Phys. Chem. Chem. Phys, 2016, 18, 19050-19061.

54 A. Caspi, R. Granek and M. Elbaum, Phys. Rev. Lett., 2000, 85, 5655. 